\title{
Hydrostatic pressure Exerts Different Effects on Community Structure and Metabolic Capacities of Marine Particle-Attached and Free-Living Microorganisms in Decomposition of the Sinking Particles

\author{
YUANQI XU ${ }^{1}$, JIANGYAN LI ${ }^{1}$, JIAHUA WANG ${ }^{1}$ AND \\ JIASONG FANG ${ }^{2}$
} \\ ${ }^{1}$ College of Marine Sciences, Shanghai Ocean University \\ ${ }^{2}$ Shanghai Ocean University \\ Presenting Author: lancy_x@126.com
}

Heterotrophic microorganisms form the ecological dichotomy in the ocean, in either particle-attached (PAM) or free-living (FLM) lifestyle, and play a dominant role in the global ocean carbon cycle. However, the relative impact of the two microbial groups in mediating decomposition of particulate organic matter (POM) and degradation of dissolved organic matter (DOM) is not well understood. In this study, ${ }^{13} \mathrm{C}$-labeled POM produced by Thalassiosira Weissflogii was used as substrate to incubate PAM and FLM assemblages. The surface water of Mariana Trench was inoculated with ${ }^{13} \mathrm{C}$-labeled and non labeledPOM under hydrostatic pressure of $0.1,10,30,60$ and $100 \mathrm{MPa}$ in order to simulate the sinking particles from the surface to the deep water and to investigate the interactions between PAM and FLM and their contributions to POM decomposition and degradation.. Our results showed that the effects of PAM and FLM in POM decomposition varied substantially under different hydrostatic pressure. DNA-stable isotope probing (SIP) and high-throughput sequencing of $16 \mathrm{~S}$ rRNA genes showed that 22 active OTUs dominate POM decomposition and degradation. Proteomics analysis revealed that PAM and FLM showed contrasting patterns in gene expression for motility, flagelin and $A B C$ transporter. Our results significantly expanded the understanding on how pressure affects community structure and metabolic function of microbes associated with the descending particulates in the ocean. 